\title{
Synthesis, Antimicrobial Activity and in silico Studies on Eugenol Eters
}

\section{Jelena Lazarević, $1,{ }^{*}$ Ana Kolarević, ${ }^{2}$ Gordana Stojanović,,${ }^{3,}$ Andrija Šmelcerović, ${ }^{1}$ Pierangela Ciuffreda ${ }^{4}$ and Enzo Santaniello ${ }^{5}$}

\author{
${ }^{1}$ Department of Chemistry, Faculty of Medicine, University of Niš, Bul. dr Zorana Đinđića 81, 18000 Niš, Serbia \\ ${ }^{2}$ Department of Pharmacy, Faculty of Medicine, University of Niš, Bul. dr Zorana Đinđića 81, 18000 Niš, Serbia \\ ${ }^{3}$ Department of Chemistry, Faculty of Science and Mathematics, University of Niš, Višegradska 33, 18000 Niš, Serbia \\ ${ }^{4}$ Dipartimento di Scienze Biomediche e Cliniche "L. Sacco", Università degli Studi di Milano, \\ Via G.B. Grassi 74, 20157 Milano, Italy
}

${ }^{5}$ Department of Biomedical Sciences, Humanitas University, Via Manzoni 113, 20089 Rozzano - Milano, Italy

*Corresponding author: E-mail: E-mail: jelena217@yahoo.com (JL)and gocast@pmf.ni.ac.rs (GS)

Received: 28-03-2018

\begin{abstract}
The results presented herein represent our continued study based on the modification of phenolic functionality in molecules originated from natural sources by acylation. A small focused library of nineteen eugenyl esters, with four of which are new compounds, is reported. All compounds were subjected to in vitro antimicrobial testing. In silico studies were carried out calculating physico-chemical, pharmacokinetic and toxicological properties, providing more data as additional guidance for further research.
\end{abstract}

Keywords: Chemical synthesis; eugenyl esters; in vitro antimicrobial activity; in silico calculation

\section{Introduction}

Natural products, especially those derived from plants, represent an excellent source of new bioactive compounds since their structures may be used as natural pharmacophores enabling structural derivatization. ${ }^{1}$ Phenylpropenes belong to a vast family of compounds called phenylpropanoids that are biosynthesized in plants from the amino acids phenylalanine and tyrosine via shikimate pathway. ${ }^{2}$ Phenylpropenes, such as eugenol, isoeugenol, chavicol and anethole are biosynthesized by flowering plants as defense compounds against animals and microorganisms. ${ }^{3}$ Because of the antimicrobial properties of phenylpropenes, as well as their pleasant aromas and flavors, since ancient times humans have used plant materials containing such compounds to preserve and flavor their food (cloves, allspice, black pepper, anise, fennel; all of these containing significant amounts of phenylpropenes) and as medicinal agents. ${ }^{3}$

Eugenol (4-allyl-2-methoxyphenol) is naturally occurring in essential oils of variety of plants belonging to
Lamiaceae, Lauraceae, Myrtaceae and Myristicaceae families. It is one of the major constituents of clove (Syzygium aromaticum (L.) Merrill \& Perry, Myrtaceae) oil and is largely used both in foods and as flavouring agent. ${ }^{4,5}$ Eugenol and its close analogues have several proven biological activities $^{6-16}$ which make it useful in pharmaceutical production. Additionally, eugenol has been described as an agent with dual effect: antioxidant and pro-oxidant, postulating the existence of beneficial properties in both prevention of cancer formation and in cancer treatment. ${ }^{17}$ According to previous in vitro and in vivo studies eugenol might function as a tumor suppressor in most cancer types inspected. ${ }^{17-20}$

The activity of eugenol against microorganisms is also well known. ${ }^{21,22}$ The mechanisms of antimicrobial action have been documented in both bacteria and fungi. ${ }^{21,23-25}$ According to a review discussing mechanisms of antimicrobial action of eugenol and essential oils containing eugenol, two main uses of eugenol are particularly indicated: against food-decaying microorganisms and 
against bacteria causing oral clinical conditions. ${ }^{21}$ In a review published by Freires et al. ${ }^{26}$ it was concluded that due to its promising antimicrobial activity against streptococci, eugenol should be considered as an anti-cariogenic agent worth of further clinical testing. The mode of action of eugenol against microbials has been thoroughly investigated and reviewed by Marchese $e t$ al. ${ }^{21}$ In this paper a systematic presentation of the mechanisms against bacteria and fungi known so far is given. Compounds obtained by derivatisation of eugenol have also been reported as antimicrobial agents..$^{1,27-30}$ So far, the most frequently investigated eugenol derivatives in bioassays are glycosides ${ }^{27-30}$ and esters. ${ }^{31-33}$ Like eugenol, eugenyl esters can also represent compounds of promising biological properties. Several papers on isolation and/or synthesis and biological activity of eugenyl esters have reported wide range of activities: antioxidant, ${ }^{9,11,13,34}$ anticancer, ${ }^{35}$ larvicidal, ${ }^{37}$ anti-leishmaniasis. ${ }^{38}$ On the other hand, little data exists on antimicrobial activity, reporting only few compounds involved with respect to the total number synthesized. Antimicrobial assays evaluated antibacterial activity of eugenyl esters of acetic, 3-methylbutanoic, pentanoic and benzoic acids, ${ }^{31-33}$ but no data related to antifungal activity of such derivatives was reported.

The diverse pharmacological activity of eugenol and eugenyl esters, scarce results on antibacterial and no results on antifungal activity of ester derivatives, have determined the aim of our study. By making a modification of a phenolic functionality in eugenol, we have obtained a series of ester compounds (3a-s), performed their structural characterization, in vitro antimicrobial testing and in silico calculation of physico-chemical, pharmacokinetic and toxicological properties. An important contribution of this study is the synthesis of four new compounds $(3 \mathbf{l}, \mathbf{3 n}, \mathbf{3 p}$ and $\mathbf{3 r}$ ), followed by the results obtained in antibacterial together with the first results ever obtained in an antifungal assay, and by in silico calculations. All together represent an important aspect to guide further research.

\section{Materials and Methods}

\section{1. Chemicals Used}

All the reagents, standards and solvents used were of analytical reagent grade. Unless specified otherwise, all chemicals were purchased from Merck (Darmstadt, Germany).

\section{2. General Synthetic Procedures}

Acetyl, benzoyl and palmitoyl chloride (2a, 2r, 2s) were purchased from Sigma-Aldrich. For the preparation of acyl chlorides $\mathbf{2} \mathbf{b}-\mathbf{p}, \mathbf{2} \mathbf{r}$ and of eugenyl esters (Table 1 , entries 3a-s) methods from the literature ${ }^{39-42}$ were utilized and general procedures for the synthesis as reported in our previously published paper was followed..$^{43}$ Scheme 1 represents the synthesis of eugenyl esters.

The obtained esters of eugenol 3a-s were purified by column chromatography, stationary phase Silica Gel 60 (70-230 mesh), mobile phase (hexane/diethyl ether, gradient 9:1 to 6:4). For yields see Table 1. For solid compounds 3q, $3 \mathbf{r}$ and $3 \mathbf{s}$ melting points were determined in a Stuart Scientific SMP3 apparatus and are uncorrected.

\section{Eugenyl Acetate (3a)}

Chromatographic purification gave yellowish oil. $\mathrm{C}_{12} \mathrm{H}_{14} \mathrm{O}_{3}(M=206.24)$; yield 96\%; MS (EI): $\mathrm{m} / z$ (\%) 206 $\left(\mathrm{M}^{+}\right), 164$ (100), 149 (27.23), 133 (15.32), 131 (20.72), 121 (11.81), 104 (16.72), 103 (18.52), 91 (23.22), 77 (17.02), 43 (14.18); RI (HP5-MS): $1538 ;{ }^{1} \mathrm{H}$ NMR $(500.13 \mathrm{MHz}$, $\left.\mathrm{CDCl}_{3}\right): \delta 6.98(1 \mathrm{H}, \mathrm{d}, J=8 \mathrm{~Hz}, \mathbf{H}-6), 6.77(2 \mathrm{H}, \mathrm{m}, \mathbf{H}-3$ and H-5), $6.04(1 \mathrm{H}, \mathrm{m}, \mathbf{H}-9), 5.13(2 \mathrm{H}, \mathrm{m}, \mathbf{H}-\mathbf{1 0}), 3.85$ (3H, s, H-7), $3.41(2 \mathrm{H}, \mathrm{d}, J=6 \mathrm{~Hz}, \mathbf{H}-8), 2.34(3 \mathrm{H}, \mathrm{s}, \mathbf{H}-12)$; ${ }^{13} \mathrm{C}$ NMR $\left(125.76 \mathrm{MHz}, \mathrm{CDCl}_{3}\right): \delta 169.28$ (C-11), 150.88 (C-2), 139.05 (C-1), 138.00 (C-4), 137.06 (C-9), 122.53 (C6), 120.69 (C-5), 116.19 (C-10), 112.73 (C-3), 55.84 (C-7), 40.12 (C-8), 20.71 (C-12). Physical and spectroscopic data were in accordance with those reported. ${ }^{37,38}$

\section{Eugenyl Propanoate (3b)}

Chromatographic purification gave yellowish oil. $\mathrm{C}_{13} \mathrm{H}_{16} \mathrm{O}_{3}(M=220.26)$; yield $89 \%$; $\mathrm{MS}(\mathrm{EI}): \mathrm{m} / z$ (\%) 220 $\left(\mathrm{M}^{+}\right), 165$ (16.52), 164 (100), 149 (23.02), 133 (12.01), 132 (9.11), 131 (13.91), 104 (10.11), 103 (10.51), 91 (13.21), 57 (14.01); RI (HP5-MS): $1635 ;{ }^{1} \mathrm{H}$ NMR $(500.13 \mathrm{MHz}$, $\left.\mathrm{CDCl}_{3}\right): \delta 6.98(1 \mathrm{H}, \mathrm{d}, J=8 \mathrm{~Hz}, \mathbf{H}-6), 6.70-6.90(2 \mathrm{H}, \mathrm{m}$, H-3 and H-5), 5.90-6.07 (1H, m, H-9), 5.04-5.23 (2H, m, H-10), $3.84(3 \mathrm{H}, \mathrm{s}, \mathrm{H}-7), 3.41(2 \mathrm{H}, \mathrm{d}, J=6 \mathrm{~Hz}, \mathbf{H}-8), 2.64$ $(2 \mathrm{H}, \mathrm{q}, J=8 \mathrm{~Hz}, \mathbf{H}-\mathbf{1 2}), 1.31(3 \mathrm{H}, \mathrm{t}, J=7 \mathrm{~Hz}, \mathbf{H}-\mathbf{1 3}) ;{ }^{13} \mathrm{C}$ NMR (125.76 MHz, $\left.\mathrm{CDCl}_{3}\right): \delta 172.72(\mathbf{C}-11), 150.96(\mathbf{C}-$ 2), 138.87 (C-1), 138.13 (C-4), 137.13 (C-9), 122.55 (C-6), 120.68 (C-5), 116.14 (C-10), 112.75 (C-3), 55.84 (C-7), 40.12 (C-8), 27.39 (C-12), 9.23 (C-13). Physical and spectroscopic data were in accordance with those reported. ${ }^{37,38}$

\section{Eugenyl Butanoate (3c)}

Chromatographic purification gave colorless oil. $\mathrm{C}_{14} \mathrm{H}_{18} \mathrm{O}_{3}(M=234.29)$; yield 87\%; MS (EI): $m / z(\%) 234$ $\left(\mathrm{M}^{+}\right), 165$ (10.91), 164 (100), 149 (19.02), 133 (10.01), 132 (7.91), 131 (11.21), 104 (8.21), 103 (8.41), 91 (10.31), 43 (8.41); RI (HP5-MS): $1729 ;{ }^{1} \mathrm{H}$ NMR $(500.13 \mathrm{MHz}$, $\left.\mathrm{CDCl}_{3}\right): \delta 6.97(1 \mathrm{H}, \mathrm{d}, J=8 \mathrm{~Hz}, \mathbf{H}-6), 6.77-6.84(2 \mathrm{H}, \mathrm{m}$, H-3 and H-5), 5.94-6.04 (1H, m, H-9), 5.09-5.17 (2H, m, H-10), 3.83 (3H, s, H-7), 3.41 (2H, d, J = 6 Hz, H-8), 2.59 $(2 \mathrm{H}, \mathrm{t}, J=7 \mathrm{~Hz}, \mathbf{H}-\mathbf{1 2}), 1.83(2 \mathrm{H}, \mathrm{spt}, J=7 \mathrm{~Hz}, \mathbf{H}-\mathbf{1 3}), 1.08$ $(3 \mathrm{H}, \mathrm{t}, J=7 \mathrm{~Hz}, \mathbf{H}-\mathbf{1 4}) ;{ }^{13} \mathrm{C}$ NMR $\left(125.76 \mathrm{MHz}, \mathrm{CDCl}_{3}\right): \delta$ 171.89 (C-11), 150.93 (C-2), 138.87 (C-1), 138.09 (C-4), 137.13 (C-9), 122.57 (C-6), 120.68 (C-5), 116.13 (C-10), 112.73 (C-3), 55.81 (C-7), 40.12 (C-8), 35.94 (C-12), 18.62 (C-13), 13.62 (C-14). Physical and spectroscopic data were in accordance with those reported. ${ }^{11,38}$ 


\section{Eugenyl 2-Methylpropanoate (3d)}

Chromatographic purification gave colorless oil. $\mathrm{C}_{14} \mathrm{H}_{18} \mathrm{O}_{3}(M=234.29)$; yield $76 \%$; MS (EI): $m / z(\%) 234$ $\left(\mathrm{M}^{+}\right), 165$ (11.11), 164 (100), 149 (16.72), 133 (8.71), 131 (10.01), 103 (8.71), 91 (10.61), 77 (7.71), 71 (7.81), 43 (8.41); RI (HP5-MS): $1679 ;{ }^{1} \mathrm{H}$ NMR $(500.13 \mathrm{MHz}$, $\left.\mathrm{CDCl}_{3}\right): \delta 6.94-7.03(1 \mathrm{H}, \mathrm{m}, \mathbf{H}-6), 6.77-6.82(2 \mathrm{H}, \mathrm{m}, \mathbf{H}-\mathbf{3}$ and $\mathbf{H}-5), 5.95-6.03(1 \mathrm{H}, \mathrm{m}, \mathbf{H}-9), 5.10-5.16(2 \mathrm{H}, \mathrm{m}$, H-10), 3.83 (3H, s, H-7), $3.41(2 \mathrm{H}, \mathrm{d}, J=6.24 \mathrm{~Hz}, \mathbf{H}-8)$, $2.86(2 \mathrm{H}, \mathrm{spt}, J=6.9 \mathrm{~Hz}, \mathrm{H}-12), 1.35(6 \mathrm{H}, \mathrm{d}, J=6.9 \mathrm{~Hz}, 2 \times$ $\mathrm{CH}_{3}: \mathbf{H}-\mathbf{1 3}$ and $\left.\mathbf{H}-\mathbf{1 4}\right) ;{ }^{13} \mathrm{C}$ NMR $\left(125.76 \mathrm{MHz}, \mathrm{CDCl}_{3}\right): \delta$ 175.40 (C-11), 150.97 (C-2), 138.76 (C-1), 138.25 (C-4), 137.16 (C-9), 122.49 (C-6), 120.68 (C-5), 116.08 (C-10), 112.80 (C-3), 55.87 (C-7), 40.11 (C-8), 34.00 (C-12), 19.08 $\left(2 \times \mathrm{CH}_{3}\right.$ : C-13 and C-14). Physical and spectroscopic data were in accordance with those reported. ${ }^{33,44}$

\section{Eugenyl Pentanoate (3e)}

Chromatographic purification gave colorless oil. $\mathrm{C}_{15} \mathrm{H}_{20} \mathrm{O}_{3}(M=248.32)$; yield $85 \%$; MS (EI): $m / z(\%) 248$ $\left(\mathrm{M}^{+}\right), 165$ (11.01), 164 (100), 149 (15.82), 133 (8.81), 131 (9.61), 104 (7.41), 103 (7.51), 91 (9.31), 57 (14.61), 55 (6.71); RI (HP5-MS): 1829 ; ${ }^{1} \mathrm{H}$ NMR $(500.13 \mathrm{MHz}$, $\left.\mathrm{CDCl}_{3}\right): \delta 6.96(1 \mathrm{H}, \mathrm{d}, J=8 \mathrm{~Hz}, \mathrm{H}-6), 6.76-6.83(2 \mathrm{H}, \mathrm{m}$, H-3 and H-5), 5.94-6.03 (1H, m, H-9), 5.09-5.16 (2H, m, H-10), 3.83 (3H, s, H-7), $3.40(2 \mathrm{H}, \mathrm{d}, J=6 \mathrm{~Hz}, \mathbf{H}-8), 2.60$ $(2 \mathrm{H}, \mathrm{t}, J=8 \mathrm{~Hz}, \mathbf{H}-\mathbf{1 2}), 1.78(2 \mathrm{H}$, quin, $J=7 \mathrm{~Hz}, \mathbf{H}-\mathbf{1 3})$, $1.49(2 \mathrm{H}, \mathrm{sxt}, J=7 \mathrm{~Hz}, \mathbf{H}-\mathbf{1 4}), 1.0(3 \mathrm{H}, \mathrm{t}, J=7 \mathrm{~Hz}, \mathbf{H}-\mathbf{1 5})$; ${ }^{13} \mathrm{C}$ NMR $\left(125.76 \mathrm{MHz}, \mathrm{CDCl}_{3}\right): \delta 172.07$ (C-11), 150.93 (C-2), 138.86 (C-1), 138.09 (C-4), 137.13 (C-9), 122.55 (C6), 120.68 (C-5), 116.13 (C-10), 112.73 (C-3), 55.81 (C-7), 40.11 (C-8), 33.79 (C-12), 27.13 (C-13), 22.22 (C-14), $13.76(\mathbf{C}-15)$. Physical and spectroscopic data were in accordance with those reported. ${ }^{11,38}$

\section{Eugenyl 3-Methylbutanoate (3f)}

Chromatographic purification gave colorless oil. $\mathrm{C}_{15} \mathrm{H}_{20} \mathrm{O}_{3}(M=248.32)$; yield $85 \%$; MS (EI): $m / z$ (\%) 248 $\left(\mathrm{M}^{+}\right)$(3.20), 165 (11.41), 164 (100), 149 (15.72), 133 (7.41), 132 (6.81), 121 (2.90); RI (HP5-MS): 1785; ${ }^{1} \mathrm{H}$ NMR $\left(500.13 \mathrm{MHz}, \mathrm{CDCl}_{3}\right): \delta 6.96(1 \mathrm{H}, \mathrm{d}, J=6.3 \mathrm{~Hz}, \mathbf{H}-6)$, 6.78-6.82 (2H, m, H-3 and H-5), 5.98 (1H, s, H-9), 5.10$5.16(2 \mathrm{H}, \mathrm{m}, \mathbf{H}-10), 3.83(3 \mathrm{H}, \mathrm{s}, \mathbf{H}-7), 3.41(2 \mathrm{H}, \mathrm{d}, J=6.24$ $\mathrm{Hz}, \mathbf{H}-8), 2.48(2 \mathrm{H}, \mathrm{d}, J=7.63 \mathrm{~Hz}, \mathbf{H}-12), 2.29(1 \mathrm{H}, \mathrm{m}$, H-13), $1.10\left(6 \mathrm{H}, \mathrm{d}, J=6.24 \mathrm{~Hz}, 2 \times \mathrm{CH}_{3}\right.$ : H-14 and H-15); ${ }^{13} \mathrm{C}$ NMR $\left(125.76 \mathrm{MHz}, \mathrm{CDCl}_{3}\right): \delta 171.26(\mathbf{C}-11), 150.94$ (C-2), 138.87 (C-1), 138.08 (C-4), 137.13 (C-9), 122.57 (C6), 120.68 (C-5), 116.11 (C-10), 112.75 (C-3), 55.75 (C-7), 43.14 (C-12), 40.11 (C-8), 25.97 (C-13), $22.39\left(2 \times \mathrm{CH}_{3}\right.$ : $\mathrm{C}-14$ and $\mathrm{C}-15)$. Physical and spectroscopic data were in accordance with those reported. ${ }^{33}$

\section{Eugenyl Hexanoate (3g)}

Chromatographic purification gave colorless oil. $\mathrm{C}_{16} \mathrm{H}_{22} \mathrm{O}_{3}(M=262.34)$; yield 82\%; MS (EI): $m / z(\%) 262$ $\left(\mathrm{M}^{+}\right), 165$ (10.71), 164 (100), 149 (13.31), 133 (7.11), 132
(6.11), 103 (6.71), 91 (7.91), 55 (6.81), 43 (8.71); RI (HP5MS): $1932 ;{ }^{1} \mathrm{H}$ NMR $\left(500.13 \mathrm{MHz}, \mathrm{CDCl}_{3}\right): \delta 6.94(1 \mathrm{H}, \mathrm{d}$, $J=8 \mathrm{~Hz}, \mathbf{H}-\mathbf{6}), 6.72-6.83(2 \mathrm{H}, \mathrm{m}, \mathbf{H}-\mathbf{3}$ and $\mathbf{H}-\mathbf{5}), 5.90-6.03$ (1H, m, H-9), 5.05-5.17 (2H, m, H-10), 3.82 (3H, s, H-7), $3.39(\mathrm{~d}, J=6.6 \mathrm{~Hz}, 2 \mathrm{H}, \mathbf{H}-8), 2.59(2 \mathrm{H}, \mathrm{t}, J=7.5 \mathrm{~Hz}, \mathbf{H}-12)$, $1.76(2 \mathrm{H}$, quin, $J=7.5 \mathrm{~Hz}, \mathbf{H}-13), 1.47(2 \mathrm{H}, \mathrm{sxt}, J=7.5 \mathrm{~Hz}$, H-15), $0.98(3 \mathrm{H}, \mathrm{t}, J=7.5 \mathrm{~Hz}, \mathbf{H}-16) ;{ }^{13} \mathrm{C}$ NMR $(125.76$ $\mathrm{MHz}, \mathrm{CDCl}_{3}$ ): $\delta 172.01$ (C-11), 150.87 (C-2), 138.80 (C1), 138.05 (C-4), 137.06 (C-9), 122.50 (C-6), 120.62 (C-5), 116.07 (C-10), 112.69 (C-3), 55.76 (C-7), 40.06 (C-8), 33.75 (C-12), 27.08 (C-13), 22.47 (C-14), 22.37 (C-15), 13.98 (C-16). Physical and spectroscopic data were in accordance with those reported. ${ }^{38}$

\section{Eugenyl Heptanoate (3h)}

Chromatographic purification gave colorless oil. $\mathrm{C}_{17} \mathrm{H}_{24} \mathrm{O}_{3}(M=276.37)$; yield $82 \%$; MS (EI): $m / z(\%) 276$ $\left(\mathrm{M}^{+}\right), 165$ (11.31), 164 (100), 149 (11.71), 133 (6.01), 132 (5.21), 131 (6.61), 103 (4.90), 91 (6.01), 55 (5.91), 43 (6.51); RI (HP5-MS): 2037; ${ }^{1} \mathrm{H}$ NMR $\left(500.13 \mathrm{MHz}, \mathrm{CDCl}_{3}\right): \delta$ $6.96(1 \mathrm{H}, \mathrm{d}, J=8 \mathrm{~Hz}, \mathbf{H}-6), 6.76-6.83(2 \mathrm{H}, \mathrm{m}, \mathbf{H}-3$ and H-5), 5.94-6.03 (1H, m, H-9), 5.09-5.16 (2H, m, H-10), $3.83(3 \mathrm{H}, \mathrm{s}, \mathbf{C}-7), 3.40(2 \mathrm{H}, \mathrm{d}, J=7 \mathrm{~Hz}, \mathbf{H}-8), 2.60(2 \mathrm{H}, \mathrm{t}, J$ $=7 \mathrm{~Hz}, \mathbf{H}-12), 1.78(2 \mathrm{H}$, quin, $J=7 \mathrm{~Hz}, \mathbf{H}-13), 1.46(2 \mathrm{H}$, bs, H-14), 1.31-1.42 (4H, m, H-15 and H-16), $0.94(3 \mathrm{H}$, bs, H-17); ${ }^{13} \mathrm{C}$ NMR $\left(125.76 \mathrm{MHz}, \mathrm{CDCl}_{3}\right): \delta 172.08(\mathbf{C}-$ 11), 150.93 (C-2), 138.86 (C-1), 138.09 (C-4), 137.13 (C9), 122.55 (C-6), 120.68 (C-5), 116.13 (C-10), 112.73 (C3), 55.81 (C-7), 40.12 (C-8), 34.10 (C-12), 31.49 (C-15), 28.75 (C-14), 25.05 (C-13), 22.55 (C-16), 14.06 (C-17). Physical and spectroscopic data were in accordance with those reported. ${ }^{38}$

\section{Eugenyl Octanoate (3i)}

Chromatographic purification gave yellowish oil. $\mathrm{C}_{18} \mathrm{H}_{26} \mathrm{O}_{3}(M=290.40)$; yield $86 \%$; MS (EI): $m / z(\%) 290$ $\left(\mathrm{M}^{+}\right), 165$ (16.52), 164 (100), 149 (10.31), 133 (5.41), 132 (4.60), 131 (5.81), 103 (4.60), 91 (5.21), 57 (10.21), 55 (7.71); RI (HP5-MS): 2141; ${ }^{1} \mathrm{H}$ NMR $(500.13 \mathrm{MHz}$, $\left.\mathrm{CDCl}_{3}\right): \delta 6.96(1 \mathrm{H}, \mathrm{d}, J=8.3 \mathrm{~Hz}, \mathbf{H}-6), 6.76-6.83(2 \mathrm{H}, \mathrm{m}$, H-3 and H-5), 5.94-6.03 (1H, m, H-9), 5.09-5.18 (2H, m, H-10), 3.83 (3H, s, C-7), $3.40(2 \mathrm{H}, \mathrm{d}, J=6.2 \mathrm{~Hz}, \mathbf{H}-8), 2.59$ $(2 \mathrm{H}, \mathrm{t}, J=7.3 \mathrm{~Hz}, \mathbf{H}-\mathbf{1 2}), 1.79(2 \mathrm{H}$, quin, $J=7.3 \mathrm{~Hz}, \mathbf{H}-\mathbf{1 3})$, 1.28-1.49 (8H, bm, H-14-H-17), 0.88-0.97 (3H, m, H-18); ${ }^{13} \mathrm{C}$ NMR (125.76 MHz, $\left.\mathrm{CDCl}_{3}\right): \delta 172.08$ (C-11), 150.93 (C-2), 138.86 (C-1), 138.09 (C-4), 137.13 (C-9), 122.57 (C-6), 120.68 (C-5), 116.13 (C-10), 112.73 (C-3), 55.81 (C-7), 40.12 (C-8), 34.10 (C-12), 31.73 (C-16), 29.05 (C-15), 28.97 (C-14), 25.10 (C-13), 22.64 (C-17), 14.11 (C-18). Physical and spectroscopic data were in accordance with those reported. ${ }^{38}$

\section{Eugenyl Nonanoate (3j)}

Chromatographic purification gave yellowish oil. $\mathrm{C}_{19} \mathrm{H}_{28} \mathrm{O}_{3}(M=304.42)$; yield 76\%; MS (EI): $m / z$ (\%) 304 $\left(\mathrm{M}^{+}\right), 165$ (11.31), 164 (100), 149 (9.01), 137 (1.90), 133 
(4.40), 132 (4.60), 131 (3.10), 121 (1.50), 107 (1.30), 104 (2.00); RI (HP5-MS): 2246; ${ }^{1} \mathrm{H}$ NMR (500.13 MHz, $\left.\mathrm{CDCl}_{3}\right): \delta 6.96(1 \mathrm{H}, \mathrm{d}, J=8.3 \mathrm{~Hz}, \mathbf{H}-6), 6.70-6.84(2 \mathrm{H}, \mathrm{m}$, H-3 and H-5), 5.92-6.04 (1H, m, H-9), 5.03-5.18 (2H, m, H-10), 3.83 (3H, s, C-7), 3.40 (2H, d, J=6.2 Hz, H-8), 2.59 $(2 \mathrm{H}, \mathrm{t}, J=7.3 \mathrm{~Hz}, \mathbf{H}-\mathbf{1 2}), 1.78(2 \mathrm{H}$, quin, $J=7.5 \mathrm{~Hz}, \mathbf{H}-\mathbf{1 3})$, 1.29-1.49 (10H, bm, H-14-H-18), 0.83-1.06 (3H, m, H-19); ${ }^{13} \mathrm{C}$ NMR (125.76 MHz, $\mathrm{CDCl}_{3}$ ): $\delta 172.08$ (C-11), 150.93 (C-2), 138.86 (C-1), 138.09 (C-4), 137.13 (C-9), 122.57 (C-6), 120.68 (C-5), 116.13 (C-10), 112.73 (C-3), 55.81 (C-7), 40.11 (C-8), 34.10 (C-12), 31.84 (C-17), 29.27 (C-16), 29.19 (C-15), 29.10 (C-14), 25.09 (C-13), 22.68 (C-18), 14.13 (C-19). Physical and spectroscopic data were in accordance with those reported. ${ }^{38}$

\section{Eugenyl Decanoate (3k)}

Chromatographic purification gave colorless oil. $\mathrm{C}_{20} \mathrm{H}_{30} \mathrm{O}_{3}(M=318.45)$; yield 85\%; MS (EI): $m / z(\%) 318$ $\left(\mathrm{M}^{+}\right), 166$ (1.00), 165 (11.31), 164 (100), 149 (7.91), 147 (1.00), 137 (1.70), 133 (4.40), 105 (1.50), 103 (4.00); 85 (1.40); RI (HP5-MS): $2348 ;{ }^{1} \mathrm{H}$ NMR (500.13 MHz, $\left.\mathrm{CDCl}_{3}\right)$ : $\delta 6.96(1 \mathrm{H}, \mathrm{d}, J=7.6 \mathrm{~Hz}, \mathbf{H}-6), 6.78-6.82(2 \mathrm{H}, \mathrm{m}, \mathbf{H}-3$ and H-5), 5.95-6.04 (1H, m, H-9), 5.10-5.17 (2H, m, H-10), $3.83(3 \mathrm{H}, \mathrm{s}, \mathbf{C}-7), 3.40(2 \mathrm{H}, \mathrm{d}, J=6.2 \mathrm{~Hz}, \mathbf{H}-8), 2.59(2 \mathrm{H}, \mathrm{t}$, $J=7.6 \mathrm{~Hz}, \mathbf{H}-12), 1.79$ (2H, quin, $J=7.6 \mathrm{~Hz}, \mathbf{H}-\mathbf{1 3}), 1.27-$ 1.48 (12H, bm, H-14-H-19), 0.92 (3H, t, $J=6.9 \mathrm{~Hz}, \mathbf{H}-20)$; ${ }^{13} \mathrm{C} \mathrm{NMR}\left(125.76 \mathrm{MHz}, \mathrm{CDCl}_{3}\right): \delta 172.08$ (C-11), 150.93 (C-2), 138.86 (C-1), 138.09 (C-4), 137.13 (C-9), 122.57 (C6), 120.68 (C-5), 116.13 (C-10), 112.73 (C-3), 55.81 (C-7), 40.12 (C-8), 34.10 (C-12), 31.91 (C-18), 29.5 (C-16), 29.32 $\left(2 \times \mathrm{CH}_{2}: \mathbf{C}-15\right.$ and C-17), 29.10 (C-14), 25.10 (C-13), 22.71 (C-19), 14.14 (C-20). Physical and spectroscopic data were in accordance with those reported. ${ }^{38}$

\section{Eugenyl Undecanaote (31)}

Chromatographic purification gave colorless oil. $\mathrm{C}_{21} \mathrm{H}_{32} \mathrm{O}_{3}(M=332.48)$; yield 87\%; MS (EI): $m / z(\%) 332$ $\left(\mathrm{M}^{+}\right), 165$ (11.21), 164 (100), 149 (7.31), 133 (3.90), 131 (4.50), 103 (3.70), 91 (4.30), 57 (5.91), 55 (10.01); RI (HP5MS): $2449 ;{ }^{1} \mathrm{H}$ NMR $\left(500.13 \mathrm{MHz}, \mathrm{CDCl}_{3}\right): \delta 6.96(1 \mathrm{H}, \mathrm{d}$, $J=8.3 \mathrm{~Hz}, \mathbf{H}-6), 6.78-6.82(2 \mathrm{H}, \mathrm{m}, \mathbf{H}-3$ and H-5), 5.956.03 (1H, m, H-9), 5.10-5.16 (2H, m, H-10), 3.83 (3H, s, C-7), $3.40(2 \mathrm{H}, \mathrm{d}, J=6.24 \mathrm{~Hz}, \mathbf{H}-8), 2.59(2 \mathrm{H}, \mathrm{t}, J=7.28$ $\mathrm{Hz}, \mathbf{H}-12), 1.78$ (2H, quin, $J=7.28 \mathrm{~Hz}, \mathbf{H}-13), 1.28-1.45$ (14H, bm, H-14-H-20), $0.92(3 \mathrm{H}, \mathrm{t}, J=6.6 \mathrm{~Hz}, \mathbf{H}-2 \mathbf{1}) ;{ }^{13} \mathrm{C}$ NMR (125.76 MHz, $\mathrm{CDCl}_{3}$ ): $\delta 172.08$ (C-11), 150.93 (C2), 138.86 (C-1), 138.09 (C-4), 137.13 (C-9), 122.55 (C-6), 120.68 (C-5), 116.13 (C-10), 112.73 (C-3), 55.81 (C-7), 40.12 (C-8), 34.10 (C-12), 31.94 (C-19), 29.61 (C-16), 29.54 (C-17), 29.35 (C-15), 29.32 (C-18), 29.10 (C-14), 25.10 (C-13), 22.72 (C-20), 14.14 (C-21).

\section{Eugenyl Dodecanaote (3m)}

Chromatographic purification gave colorless oil. $\mathrm{C}_{22} \mathrm{H}_{34} \mathrm{O}_{3}(M=346.50)$; yield 84\%; MS (EI): $m / z$ (\%) 346 $\left(\mathrm{M}^{+}\right), 165$ (11.31), 164 (100), 149 (6.41), 133 (3.40), 131
(3.90), 103 (3.10), 91 (3.20), 57 (5.01), 55 (8.51); RI (HP5MS): $2553 ;{ }^{1} \mathrm{H}$ NMR (500.13 MHz, $\left.\mathrm{CDCl}_{3}\right): \delta 6.96(1 \mathrm{H}, \mathrm{d}, J$ $=8.3 \mathrm{~Hz}, \mathbf{H}-6), 6.77-6.82(2 \mathrm{H}, \mathrm{m}, \mathbf{H}-3$ and $\mathbf{H}-5), 5.95-6.03$ (1H, m, H-9), 5.10-5.16 (2H, m, H-10), 3.83 (3H, s, C-7), $3.40(2 \mathrm{H}, \mathrm{d}, J=6.9 \mathrm{~Hz}, \mathbf{H}-8), 2.59(2 \mathrm{H}, \mathrm{t}, J=7.28 \mathrm{~Hz}, \mathbf{H}-\mathbf{1 2})$, $1.78(2 \mathrm{H}$, quin, $J=7.6 \mathrm{~Hz}, \mathrm{H}-13), 1.28-1.51(16 \mathrm{H}, \mathrm{bm}$, H-14-H-21), $0.92(3 \mathrm{H}, \mathrm{t}, J=6.6 \mathrm{~Hz}, \mathbf{H}-22)$; ${ }^{13} \mathrm{C}$ NMR $\left(125.76 \mathrm{MHz}, \mathrm{CDCl}_{3}\right): \delta 172.08$ (C-11), 150.93 (C-2), 138.86 (C-1), 138.09 (C-4), 137.13 (C-9), 122.55 (C-6), 120.68 (C5), 116.13 (C-10), 112.72 (C-3), 55.79 (C-7), 40.12 (C-8), 34.10 (C-12), 31.94 (C-20), $29.65\left(2 \times \mathrm{CH}_{2}\right.$ : C-16 and C-18), 29.54 (C-17), 29.37 (C-15), 29.32 (C-19), 29.10 (C-14), 25.10 (C-13), 22.72 (C-21), 14.14 (C-22). Physical and spectroscopic data were in accordance with those reported. ${ }^{18,38}$

\section{Eugenyl Tridecanaote (3n)}

Chromatographic purification gave yellowish oil. $\mathrm{C}_{23} \mathrm{H}_{36} \mathrm{O}_{3}(M=360.53)$; yield 87\%; MS (EI): $m / z(\%) 360$ $\left(\mathrm{M}^{+}\right), 164$ (100), 149 (6.51), 131 (3.10), 103 (3.10), 91 (3.11), 57 (5.10), 55 (8.40); RI (HP5-MS): 2655; ${ }^{1} \mathrm{H}$ NMR $\left(500.13 \mathrm{MHz}, \mathrm{CDCl}_{3}\right): \delta 6.97(1 \mathrm{H}, \mathrm{d}, J=8.3 \mathrm{~Hz}, \mathbf{H}-6)$, 6.78-6.82 (2H, m, H-3 and H-5), 5.95-6.04 (1H, m, H-9), 5.10-5.16 (2H, m, H-10), $3.83(3 \mathrm{H}, \mathrm{s}, \mathbf{C}-7), 3.40(2 \mathrm{H}, \mathrm{d}, J=$ $6.94 \mathrm{~Hz}, \mathbf{H}-8), 2.60(2 \mathrm{H}, \mathrm{t}, J=7.28 \mathrm{~Hz}, \mathbf{H}-\mathbf{1 2}), 1.78(2 \mathrm{H}$, quin, $J=7.3 \mathrm{~Hz}, \mathbf{H}-13), 1.27-1.54$ (16H, bm, H-14-H-22), $0.92(3 \mathrm{H}, \mathrm{t}, J=6.6 \mathrm{~Hz}, \mathbf{H}-23) ;{ }^{13} \mathrm{C} \mathrm{NMR}(125.76 \mathrm{MHz}$, $\left.\mathrm{CDCl}_{3}\right): \delta 172.04(\mathbf{C}-11), 150.94$ (C-2), 138.84 (C-1), 138.13 (C-4), 137.13 (C-9), 122.57 (C-6), 120.68 (C-5), 116.13 (C-10), 112.75 (C-3), 55.79 (C-7), 40.12 (C-8), 34.10 (C-12), 31.95 (C-21), 29.38-29.70 (4× $\mathrm{CH}_{2}$ : C-16 C-19), $29.32\left(2 \times \mathrm{CH}_{2}\right.$ : C-15 and C-20), 29.10 (C-14), 25.10 (C-13), 22.72 (C-22), 14.14 (C-23).

\section{Eugenyl Tetradecanoate (3o)}

Chromatographic purification gave yellowish oil. $\mathrm{C}_{24} \mathrm{H}_{38} \mathrm{O}_{3}(M=374.56)$; yield 79\%; MS (EI): $m / z(\%) 374$ $\left(\mathrm{M}^{+}\right), 165$ (13.01), 164 (100), 149 (5.81), 133 (3.10), 132 (2.80), 131 (3.50), 69 (2.50), 57 (3.60), 55 (5.51), 43 (2.50); RI (HP5-MS): $2753 ;{ }^{1} \mathrm{H}$ NMR (500.13 MHz, $\left.\mathrm{CDCl}_{3}\right): \delta 6.97$ $(1 \mathrm{H}, \mathrm{d}, J=8.32 \mathrm{~Hz}, \mathbf{H}-6), 6.78-6.83(2 \mathrm{H}, \mathrm{m}, \mathbf{H}-3$ and $\mathbf{H}-5)$, 5.95-6.04 (1H, m, H-9), 5.10-5.17 (2H, m, H-10), 3.83 $(3 \mathrm{H}, \mathrm{s}, \mathrm{C}-7), 3.41(2 \mathrm{H}, \mathrm{d}, J=6.24 \mathrm{~Hz}, \mathbf{H}-8), 2.60(2 \mathrm{H}, \mathrm{t}, J=$ $7.63 \mathrm{~Hz}, \mathbf{H}-12), 1.78$ (2H, m, H-13), 1.27-1.50 (18H, bm, H-14-H-23), 0.97-0.88 (3H, m, H-24); ${ }^{13} \mathrm{C}$ NMR (125.76 $\mathrm{MHz}, \mathrm{CDCl}_{3}$ ): $\delta 171.97$ (C-11), 150.97 (C-2), 138.81 (C-1), 138.16 (C-4), 137.11 (C-9), 122.57 (C-6), 120.66 (C-5), 116.08 (C-10), 112.73 (C-3), 55.76 (C-7), 40.12 (C-8), 34.08 (C-12), 31.97 (C-22), 29.56-29.72 (5 $\times \mathrm{CH}_{2}$ : C-16-C-20), 29.71 (C-18), 29.67 (C-20), 29.62 (C-17), 29.56 (C-19), 29.42 (C-22), 29.34 (C-15), 29.11 (C-14), 25.12 (C-13), 22.74 (C-23), 14.14 (C-24). Physical and spectroscopic data were in accordance with those reported. ${ }^{18}$

\section{Eugenyl Pentadecanoate (3p)}

Chromatographic purification gave colorless oil. $\mathrm{C}_{25} \mathrm{H}_{40} \mathrm{O}_{3}(M=388.58)$; yield $89 \%$; MS (EI): $m / z$ (\%) 388 
$\left(\mathrm{M}^{+}\right), 165$ (10.91), 164 (100), 149 (5.61), 131 (3.70), 69 (5.51), 57 (8.41), 55 (14.11), 43 (9.31), 41 (4.10); RI (HP5MS): 2858; ${ }^{1} \mathrm{H}$ NMR $\left(500.13 \mathrm{MHz}, \mathrm{CDCl}_{3}\right): \delta 6.97(1 \mathrm{H}, \mathrm{d}$, $J=8.32 \mathrm{~Hz}, \mathbf{H}-\mathbf{6}), 6.78-6.83(2 \mathrm{H}, \mathrm{m}, \mathbf{H}-\mathbf{3}$ and $\mathbf{H}-5)$, 5.956.04 (1H, m, H-9), 5.10-5.17 (2H, m, H-10), 3.83 (3H, s, C-7), $3.41(2 \mathrm{H}, \mathrm{d}, J=6.24 \mathrm{~Hz}, \mathbf{H}-8), 2.60(2 \mathrm{H}, \mathrm{t}, J=7.63$ $\mathrm{Hz}, \mathbf{H}-12), 1.78$ (2H, m, H-13), 1.27-1.50 (18H, bm, H-14-H-24), 0.97-0.88 (3H, m, H-25); ${ }^{13} \mathrm{C}$ NMR (125.76 $\mathrm{MHz}, \mathrm{CDCl}_{3}$ ): $\delta 172.02(\mathbf{C}-11), 150.96(\mathbf{C}-2), 138.82(\mathrm{C}-$ 1), 138.16 (C-4), 137.11 (C-9), 122.57 (C-6), 120.68 (C-5), 116.11 (C-10), 112.75 (C-3), 55.78 (C-7), 40.12 (C-8), 34.10 (C-12), 31.97 (C-23), 29.56-29.75 (6 × $\mathrm{CH}_{2}$ : C-16C21), 29.42 (C-22), 29.34 (C-15), 29.11 (C-14), 25.12 (C13), 22.74 (C-24), 14.16 (C-25).

\section{Eugenyl Hexadecanoate (3q)}

Chromatographic purification gave white solid. M.p. 39-40 ${ }^{\circ} \mathrm{C} . \mathrm{C}_{26} \mathrm{H}_{42} \mathrm{O}_{3}(M=402.61)$; yield 92\%; $\mathrm{MS}(\mathrm{EI}): \mathrm{m} / z$ (\%) $402\left(\mathrm{M}^{+}\right), 165$ (13.41), 164 (100), 149 (5.31), 133 (2.70), 132 (2.50), 131 (3.10), 69 (2.60), 57 (3.70), 55 (5.40), 43 (2.40); RI (HP5-MS): 2976; ${ }^{1} \mathrm{H}$ NMR (500.13 MHz, $\left.\mathrm{CDCl}_{3}\right): \delta 6.96(1 \mathrm{H}, \mathrm{d}, J=7.63 \mathrm{~Hz}, \mathbf{H}-6), 6.77-6.82(2 \mathrm{H}, \mathrm{m}$, H-3 and H-5), 5.95-6.04 (1H, m, H-9), 5.10-5.16 (2H, m, H-10), 3.83 (3H, s, C-7), 3.40 (2H, d, $J=6.24 \mathrm{~Hz}, \mathbf{H}-8)$, $2.60(2 \mathrm{H}, \mathrm{t}, J=7.60 \mathrm{~Hz}, \mathbf{H}-12), 1.79(2 \mathrm{H}$, quin, $J=7.50 \mathrm{~Hz}$, H-13), 1.27-1.48 (20H, bm, H-14-H-25), 0.95-0.85 (3H, m, H-26); ${ }^{13} \mathrm{C}$ NMR (125.76 MHz, $\mathrm{CDCl}_{3}$ ): $\delta 172.07$ (C11), 150.94 (C-2), 138.84 (C-1), 138.11 (C-4), 137.11 (C9), 122.55 (C-6), 120.68 (C-5), 116.11 (C-10), 112.73 (C3), 55.81 (C-7), 40.11 (C-8), 34.10 (C-12), 31.95 (C-24), 29.54-29.72 (6 $\times \mathrm{CH}_{2}$ : C-16-C-22), 29.38 (C-23), 29.32 (C-15), 29.10 (C-14), 25.10 (C-13), 22.71 (C-25), 14.14 (C-26). Physical and spectroscopic data were in accordance with those reported. ${ }^{18,38}$

\section{Eugenyl Heptadecanoate (3r)}

Chromatographic purification gave yellowish solid. M.p. $49-50{ }^{\circ} \mathrm{C}$. $\mathrm{C}_{27} \mathrm{H}_{44} \mathrm{O}_{3}(M=416.64)$; yield 76\%; MS (EI): $\mathrm{m} / z(\%) 416\left(\mathrm{M}^{+}\right), 165$ (12.11), 164 (100), 149 (4.60), 133 (2.50), 132 (2.10), 131 (2.80), 69 (3.00), 57 (4.20), 55 (6.00), 43 (3.00); RI (HP5-MS): 3096; ${ }^{1} \mathrm{H}$ NMR (500.13 MHz, $\left.\mathrm{CDCl}_{3}\right): \delta 6.97(1 \mathrm{H}, \mathrm{d}, J=8 \mathrm{~Hz}, \mathrm{H}-6), 6.74-6.88(2 \mathrm{H}, \mathrm{m}$, H-3 and H-5), 5.95-6.05 (1H, m, H-9), 5.04-5.22 (2H, m, H-10), 3.84 (3H, s, C-7), $3.42(2 \mathrm{H}, \mathrm{d}, J=7.0 \mathrm{~Hz}, \mathbf{H}-8), 2.60$ $(2 \mathrm{H}, \mathrm{t}, J=7.0 \mathrm{~Hz}, \mathbf{H}-12), 1.81(2 \mathrm{H}$, quin, $J=7.0 \mathrm{~Hz}, \mathbf{H}-\mathbf{1 3})$, 1.28-1.51 (22H, bm, H-14-H-26), $0.94(3 \mathrm{H}, \mathrm{t}, J=7.0 \mathrm{~Hz}$, H-27); ${ }^{13} \mathrm{C}$ NMR (125.76 MHz, $\mathrm{CDCl}_{3}$ ): $\delta 171.97$ (C-11), 150.97 (C-2), 138.81 (C-1), 138.16 (C-4), 137.11 (C-9), 122.57 (C-6), 120.66 (C-5), 116.10 (C-10), 112.73 (C-3), 55.76 (C-7), 40.12 (C-8), 34.10 (C-12), 31.99 (C-25), 29.57$29.77\left(7 \times \mathrm{CH}_{2}\right.$ : C-16-C-23), 29.43 (C-24), 29.35 (C-15), 29.11 (C-14), 25.12 (C-13), 22.75 (C-26), 14.14 (C-27).

\section{Eugenyl Benzoate (3s)}

Chromatographic purification gave white solid. M.p. $70-71^{\circ} \mathrm{C} . \mathrm{C}_{17} \mathrm{H}_{16} \mathrm{O}_{3}(M=268.31)$; yield $96 \%$; MS (EI): $m / z$
(\%) $268\left(\mathrm{M}^{+}\right)$(20.82), 269 (3.80), 106 (9.61), 105 (100), 91 (5.41), 78 (3.10), 77 (33.93), 65 (1.90), 51 (4.60); RI (HP5MS): $2170 ;{ }^{1} \mathrm{H}$ NMR $\left(500.13 \mathrm{MHz}, \mathrm{CDCl}_{3}\right): \delta 8.25(2 \mathrm{H}, \mathrm{d}$, $J=8.32 \mathrm{~Hz}, \mathbf{H}-2^{\prime}$ and H-6'), 7.66 (1H, t, $J=7.28 \mathrm{~Hz}, \mathbf{H}-4$ '), $7.53\left(2 \mathrm{H}, \mathrm{t}, J=7.63 \mathrm{~Hz}, \mathbf{H}-\mathbf{3}^{\prime}\right.$ and $\left.\mathbf{H}-5^{\prime}\right), 7.10(1 \mathrm{H}, \mathrm{d}, J=$ $8.32 \mathrm{~Hz}, \mathbf{H}-6), 6.87$ (1H, s, H-3), $6.85(1 \mathrm{H}, \mathrm{d}, J=8.32 \mathrm{~Hz}$, H-5), 5.98-6.06 (1H, m, H-9), 5.12-5.18 (2H, m, H-10), $3.83(3 \mathrm{H}, \mathrm{s}, \mathbf{H}-7), 3.44(2 \mathrm{H}, \mathrm{d}, J=6.94 \mathrm{~Hz}, \mathbf{H}-8) ;{ }^{13} \mathrm{C} \mathrm{NMR}$ $\left(125.76 \mathrm{MHz}, \mathrm{CDCl}_{3}\right): \delta 164.91$ (C-11), 151.13 (C-2), 139.08 (C-4), 138.22 (C-9), 137.14 (C-1)), 133.42 (C-4'), 130.32 (C-2' and C-6'), 129.55 (C-1'), 128.51 (2 $\times$ ArC: C-3' and C-5'), 122.68 (C-5), 120.76 (C-6), 116.17 (C-10), 112.88 (C-3), 55.90 (C-7), 40.15 (C-8). Physical and spectroscopic data were in accordance with those reported. ${ }^{11,37}$

\section{3. Identification of Synthetized Compounds}

\section{3. 1. GC-MS Analysis}

MS spectra of samples of the synthesized compounds were recorded on a 7890/7000B GC/MS/MS triple quadrupole system (Agilent Technologies, USA, equipped with a Combi PAL auto sampler). The fused silica capillary column HP-5MS (5\% phenylmethylsiloxane, $30 \mathrm{~m} \times 0.25$ $\mathrm{mm}$, film thickness $0.25 \mu \mathrm{m}$, Agilent Technologies, Palo Alto, CA, USA) was used. The injector, source and interface operated at 250,230 and $300{ }^{\circ} \mathrm{C}$, respectively. The temperature program: from 60 for $5 \mathrm{~min}$ isothermal to 300 ${ }^{\circ} \mathrm{C}$ at a heating rate of $8{ }^{\circ} \mathrm{C} / \mathrm{min}$ and on $300{ }^{\circ} \mathrm{C}$ for $5 \mathrm{~min}$ isothermal. The solutions in hexane were injected in split ratio 10:1. The carrier gas was helium with a flow of 1.0 $\mathrm{mL} / \mathrm{min}$. Post run: back flash for $1.89 \mathrm{~min}$, at $280^{\circ} \mathrm{C}$, with helium at $50 \mathrm{psi}$. MS conditions were as follows: ionization voltage of $70 \mathrm{eV}$, acquisition mass range 50-650, scan time $0.32 \mathrm{~s}$. Semi-quantitative analysis was carried out directly from peak areas in the GC profile.

Linear retention indices $(\mathrm{RI})$ were determined based on the retention times of $\mathrm{C}_{8}-\mathrm{C}_{40}$ alkanes run on HP-5MS column using the above mentioned temperature programme. $^{45}$

\section{3. 2. NMR Analysis}

NMR spectra were registered on a Bruker AVANCE 500 spectrometer equipped with a $5 \mathrm{~mm}$ broadband reverse probe with field $\mathrm{z}$-gradient operating at 500.13 and 125.76 MHz for ${ }^{1} \mathrm{H}$ and ${ }^{13} \mathrm{C}$, respectively. All NMR spectra were recorded at $298 \mathrm{~K}$ in $\mathrm{CDCl}_{3}$ (isotopic enrichment 99.95\%) solution. Chemical shifts are reported on the $\delta$ (ppm) scale and are relative to residual $\mathrm{CHCl}_{3}$ signals $(7.24$ for ${ }^{1} \mathrm{H}$ and $77.0 \mathrm{ppm}$, central line, for ${ }^{13} \mathrm{C}$ spectra respectively), and scalar coupling constants are reported in hertz. The experimental error in the measured ${ }^{1} \mathrm{H}-{ }^{1} \mathrm{H}$ coupling constants was $\pm 0.5 \mathrm{~Hz}$.

The signals assignment was given by a combination of $1 \mathrm{D}$ and 2D COSY and HSQC experiments, using standard Bruker pulse programs. 
Acquisition parameters for $1 \mathrm{D}$ were as follows: ${ }^{1} \mathrm{H}$ spectral width of $5000 \mathrm{~Hz}$ and $32 \mathrm{~K}$ data points providing a digital resolution of ca. $0.305 \mathrm{~Hz}$ per point, relaxation delay $2 \mathrm{~s} ;{ }^{13} \mathrm{C}$ spectral width of $29412 \mathrm{~Hz}$ and $64 \mathrm{~K}$ data points providing a digital resolution of ca. $0.898 \mathrm{~Hz}$ per point, relaxation delay $2.5 \mathrm{~s}$. The experiments were performed through standard pulse sequences. gCOSY- 45 experiments were acquired with $512 \mathrm{t} 1$ increments; $2048 \mathrm{t} 2$ points; spectral/spectrum width $10.0 \mathrm{ppm}$. The acquisition data for gHSQC and gHMBC experiments were obtained with 512 t 1 increments; 2048 t 2 points; spectral/spectrum width $10.0 \mathrm{ppm}$ for ${ }^{1} \mathrm{H}$ and $220 \mathrm{ppm}$ for ${ }^{13} \mathrm{C}$. Delay values were optimized for ${ }^{1} J_{\mathrm{C}, \mathrm{H}} 140.0 \mathrm{~Hz}$ and ${ }^{\mathrm{n}} \mathrm{J}_{\mathrm{C}, \mathrm{H}} 3.0 \mathrm{~Hz}$. Zero filling in $\mathrm{F} 1$ to $1 \mathrm{~K}, \mathrm{p} / 2$ shifted sine-bell squared (for gHSQC) or sinebell (for gHMBC) apodization functions were used for processing.

\section{4. Antimicrobial Activity}

\section{4. 1. Microbial Strains}

The in vitro antimicrobial activity of the synthesized compounds was tested against a panel of laboratory control strains belonging to the American Type Culture Collection Maryland, USA: Gram-positive: Bacillus subtilis ATCC 6633 and Staphylococcus aureus ATCC 6538; Gram-negative: Escherichia coli ATCC 8739, fungal organisms: Aspergillus niger ATCC 16404 and Candida albicans ATCC 10231. The Gram-negative bacteria Salmonella abony NCTC 6017 and Salmonela typhimurium ATCC 14028 were obtained from the National Collection of Type Cultures. All microorganisms were maintained at $-20^{\circ} \mathrm{C}$ under appropriate conditions and regenerated twice before use in the manipulations.

\section{4. 2. Screening of Antimicrobial Activity}

The minimal inhibitory concentration (MIC) of esters was determined based on a broth microdilution method in 96-well microtitre plates. ${ }^{46}$ The inocula of the bacterial strains were prepared from overnight broth cultures and suspensions were adjusted to $0.5 \mathrm{McF}$ arland standard turbidity. Dimethyl sulphoxide (10\% aqueous solution) was used to dissolve and to dilute samples to the highest concentration to be tested (stock concentrations $2 \mathrm{mg}$ / $\mathrm{mL}$ ). A serial doubling dilution of the samples was prepared in a 96-well microtiter plate, using the method of Sarker et al. ${ }^{47}$ with slight modifications. The minimal bactericidal/fungicidal concentration (MBC/MFC) was evaluated as the lowest concentration of tested samples at which inoculated microorganisms were $99.9 \%$ killed. Tests were carried out in triplicate.

\section{5. In silico Physico-chemical, Pharmacokinetic and Toxicological Properties of the Synthetized Compounds}

In order to obtain a complete picture of the synthesized compounds (3a-s) an in silico study was performed. Physico-chemical, pharmacokinetic and toxicological properties of compounds were calculated using the Molinspiration, ${ }^{48}$ admetSAR, ${ }^{49}$ DataWarrior ${ }^{50}$ and Toxtree ${ }^{51}$ prediction tools.

\section{Results and Discussion}

\section{1. Chemical Synthesis}

A small focused library of nineteen eugenyl esters was synthesized. To the best of our knowledge four out of nineteen compounds are new (3l, $\mathbf{3 n}, \mathbf{3 p}$ and $\mathbf{3 r}$; Scheme 1). All compounds were synthesized according to previously published standard methodology and with high yields ranging from $76 \%$ to $96 \%$ (Table 1 ).

\section{2. Antimicrobial Activity}

The results obtained in broth microdilution assay are given in Table 2. The assayed samples were less effective than antibiotic/antimycotic used as reference standard and if noted, activity was never greater than the values obtained for the parent compound 1 (MIC/MBC/MFC never exceeded $0.5 \mathrm{mg} / \mathrm{mL}$, Table 2). The results are indicating selective susceptibility of the microorganisms, with $B$. subtilis (3d, 3l, $\mathbf{3 n}-\mathbf{r}), S$. aureus (3e and $\mathbf{3 f}$ ), A. niger (3f, $\mathbf{3 s})$

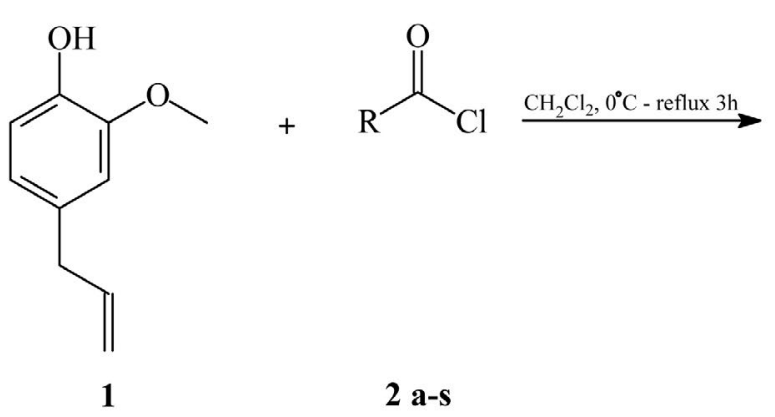

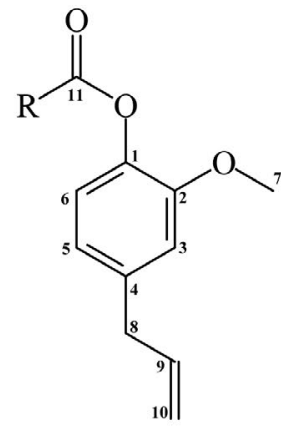

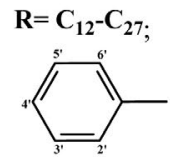

3 a-s

Scheme 1. Synthesis of eugenyl esters 
Table 1. Eugenyl esters: chemical entity, yields (\%) and entry

\begin{tabular}{lcc}
\hline $\mathbf{R}$ & Yield (\%) & Entry \\
\hline $\mathrm{CH}_{3}$ & 96 & $\mathbf{3 a}$ \\
$\mathrm{CH}_{2} \mathrm{CH}_{3}$ & 89 & $\mathbf{3 b}$ \\
$\mathrm{CH}_{2} \mathrm{CH}_{2} \mathrm{CH}_{3}$ & 87 & $\mathbf{3 c}$ \\
$\mathrm{CH}\left(\mathrm{CH}_{3}\right)_{2}$ & 76 & $\mathbf{3 d}$ \\
$\mathrm{CH}_{2}\left(\mathrm{CH}_{2}\right)_{2} \mathrm{CH}_{3}$ & 85 & $\mathbf{3 e}$ \\
$\mathrm{CH}_{2} \mathrm{CH}\left(\mathrm{CH}_{3}\right)_{2}$ & 85 & $\mathbf{3 f}$ \\
$\mathrm{CH}_{2}\left(\mathrm{CH}_{2}\right)_{3} \mathrm{CH}_{3}$ & 82 & $\mathbf{3 g}$ \\
$\mathrm{CH}_{2}\left(\mathrm{CH}_{2}\right)_{4} \mathrm{CH}_{3}$ & 82 & $\mathbf{3 h}$ \\
$\mathrm{CH}_{2}\left(\mathrm{CH}_{2}\right)_{5} \mathrm{CH}_{3}$ & 86 & $\mathbf{3 i}$ \\
$\mathrm{CH}_{2}\left(\mathrm{CH}_{2}\right)_{6} \mathrm{CH}_{3}$ & 76. & $\mathbf{3 j}$ \\
$\mathrm{CH}_{2}\left(\mathrm{CH}_{2}\right)_{7} \mathrm{CH}_{3}$ & 85 & $\mathbf{3 \mathbf { k }}$ \\
$\mathrm{CH}_{2}\left(\mathrm{CH}_{2}\right)_{8} \mathrm{CH}_{3}$ & 87 & $\mathbf{3 \mathbf { l }}$ \\
$\mathrm{CH}_{2}\left(\mathrm{CH}_{2}\right)_{9} \mathrm{CH}_{3}$ & 84 & $\mathbf{3 \mathbf { m }}$ \\
$\mathrm{CH}_{2}\left(\mathrm{CH}_{2}\right)_{10} \mathrm{CH}_{3}$ & 87 & $\mathbf{3 \mathbf { n }}$ \\
$\mathrm{CH}_{2}\left(\mathrm{CH}_{2}\right)_{11} \mathrm{CH}_{3}$ & 79 & $\mathbf{3 \mathbf { c }}$ \\
$\mathrm{CH}_{2}\left(\mathrm{CH}_{2}\right)_{12} \mathrm{CH}_{3}$ & 89 & $\mathbf{3 p}$ \\
$\mathrm{CH}_{2}\left(\mathrm{CH}_{2}\right)_{13} \mathrm{CH}_{3}$ & 92 & $\mathbf{3 q}$ \\
$\mathrm{CH}_{2}\left(\mathrm{CH}_{2}\right)_{14} \mathrm{CH}_{3}$ & 76 & $\mathbf{3 r}$ \\
$\mathrm{Ph}$ & 96 & $\mathbf{3 s}$ \\
\hline
\end{tabular}

and C. albicans (3d, 3f, 3n, $\mathbf{3 n}$ and $\mathbf{3 s}$ ) being the most sensitive strains on the synthesized derivatives. On the other hand, all three Gram-negative microorganisms tested (E. coli, S. abony and S. typhimurium) were completely resistant to synthesized compounds tested (initial concentration $2 \mathrm{mg} / \mathrm{mL}$ ).

Four of our samples (3a, 3e, 3f, 3s) are matching the samples tested in antibacterial assays so far. ${ }^{29-31}$ Eugenyl acetate (3a) was tested in a disc diffusion method on a set of seven Gram-positive and nine Gram-negative bacteria. ${ }^{30}$ The results have shown notable antimicrobial effect against all sixteen bacteria tested, with a superior performance when compared to pure eugenol. ${ }^{30}$ Even when different methodologies were employed, two crucial and completely opposed observations can be made; our results have shown that derivatives have never exceeded the activity of the parent compound and that all were inactive against Gram-negative bacteria taken into consideration. Eugenyl benzoate (3s) was tested previously in disc diffusion assay against a smaller panel of laboratory controlled bacterial strains (two Gram-positive, two Gram-nagative).

Table 2. The minimal inhibitory (MIC) and minimal bactericidal/fungicidal (MBC/MFC) concentrations $(\mathrm{mg} / \mathrm{mL})$ of the eugenol (1) and the synthetized eugenyl esters 3a-s. MIC and MBC/MFC determinations were carried out in triplicate and consistent values were obtained for each microorganism tested.

\begin{tabular}{|c|c|c|c|c|c|c|c|}
\hline \multirow[b]{3}{*}{ Cmpd. } & \multirow{2}{*}{\multicolumn{3}{|c|}{ Bacterial strains }} & \multirow{2}{*}{ Gram-negative } & \multirow[b]{3}{*}{ S. typhimurium } & \multicolumn{2}{|c|}{ Fungal strains } \\
\hline & & Gram-positive & \multirow[b]{2}{*}{ E. coli } & & & \multirow[b]{2}{*}{ A. niger } & \multirow[b]{2}{*}{ C. albicans } \\
\hline & B. subtilis & S. aureus & & S. abony & & & \\
\hline \multirow[t]{2}{*}{1} & \multirow[t]{2}{*}{$\mathrm{MIC}=0.5$} & $\mathrm{MIC}=$ & $\mathrm{MIC}=0.5$ & $\mathrm{MIC}=0.5$ & $\mathrm{MIC}=$ & $\mathrm{MIC}=$ & $\mathrm{MIC}=0.25$ \\
\hline & & $\mathrm{MBC}=0.5$ & $\mathrm{MBC}=1.0$ & $\mathrm{MBC}=1.0$ & $\mathrm{MBC}=0.5$ & $\mathrm{MFC}=0.5$ & $\mathrm{MFC}=1.0$ \\
\hline \multirow[t]{2}{*}{$3 a$} & \multirow[t]{2}{*}{ na } & \multirow[t]{2}{*}{ na } & \multirow[t]{2}{*}{ na } & \multirow[t]{2}{*}{ na } & \multirow[t]{2}{*}{ na } & \multirow[t]{2}{*}{ na } & $\mathrm{MIC}=$ \\
\hline & & & & & & & $\mathrm{MFC}=0.5$ \\
\hline $3 b$ & na & na & na & na & na & na & na \\
\hline $3 c$ & na & na & na & na & na & na & na \\
\hline $3 d$ & $\mathrm{MIC}=0.5$ & na & na & na & na & na & $\mathrm{MIC}=0.5$ \\
\hline $3 e$ & na & na & na & na & na & na & na \\
\hline $3 \mathrm{f}$ & na & $\mathrm{MIC}=0.5$ & na & na & na & $\mathrm{MIC}=0.5$ & $\mathrm{MIC}=0.5$ \\
\hline $3 g$ & na & na & na & na & na & na & na \\
\hline $3 \mathrm{~h}$ & na & na & na & na & na & na & na \\
\hline $3 \mathrm{i}$ & na & na & na & na & na & na & na \\
\hline $3 j$ & na & na & na & na & na & na & na \\
\hline $3 \mathrm{k}$ & na & na & na & na & na & na & na \\
\hline 31 & $\mathrm{MIC}=1.0$ & na & na & na & na & na & na \\
\hline $3 \mathrm{~m}$ & na & na & na & na & na & na & na \\
\hline $3 n$ & $\mathrm{MIC}=0.5$ & na & na & na & na & na & $\mathrm{MIC}=0.5$ \\
\hline 30 & $\mathrm{MIC}=1.0$ & na & na & na & na & na & na \\
\hline $3 p$ & $\mathrm{MIC}=0.5$ & na & na & na & na & na & na \\
\hline $3 q$ & $\mathrm{MIC}=0.5$ & na & na & na & na & na & na \\
\hline $3 r$ & $\mathrm{MIC}=0.5$ & na & na & na & na & na & $\mathrm{MIC}=1.0$ \\
\hline $3 \mathrm{~s}$ & na & na & na & na & na & $\mathrm{MIC}=1.0$ & $\mathrm{MIC}=1.0$ \\
\hline Doxycycline & $\mathrm{MIC}=$ & $\mathrm{MIC}=6.25$ & $\mathrm{MIC}=$ & $\mathrm{MIC}=$ & $\mathrm{MIC}=6.25$ & nt & nt \\
\hline$(\mu \mathrm{g} / \mathrm{mL})$ & $\mathrm{MBC}=1.56$ & $\mathrm{MBC}=0.78$ & $\mathrm{MBC}=0.78$ & $\mathrm{MBC}=6.25$ & $\mathrm{MBC}>50$ & & \\
\hline Nystatin & nt & nt & nt & nt & nt & $\mathrm{MIC}=$ & $\mathrm{MIC}=$ \\
\hline$(\mu \mathrm{g} / \mathrm{mL})$ & & & & & & $\mathrm{MBC}=6.25$ & $\mathrm{MBC}=0.78$ \\
\hline DMSO 10\% & & na & na & na & na & na & na na \\
\hline aqueous & & & & & & & \\
\hline solution & & & & & & & \\
\hline
\end{tabular}

na, not active; nt, not tested 
Stronger antibacterial activity of benzoate was observed compared to its unsubstituted analogue, i.e. eugenol. ${ }^{29}$ Eugenyl pentanoate (3e) and 3-methylbutanoate (3f) were tested against seventeen different bacterial strains (ATTC, clinical or food isolates) and have shown moderate antibacterial potential, with inhibitory values (MIC) ranging from 0.32 to $6.00 \mathrm{mg} / \mathrm{mL} \cdot{ }^{31}$ No noticeable difference in the susceptibility of Gram-negative and Gram-positive bacteria was observed. ${ }^{31}$ For the rest of the synthesized compounds $(\mathbf{3 b}-\mathbf{d}, \mathbf{3 g}-\mathbf{r})$ antibacterial results are reported here for the first time. For all compounds synthetized (3as) results obtained for antifungal assay are the first ever reported (Table 2).

\section{3. In silico Study}

\section{3. 1. Physico-chemical Properties of the Eugenyl Esters 3a-s}

Lipinski's rule of five has been used to evaluate drug likeness and to determine if a pharmacologically or biologically active compound has properties that would make it a likely orally active drug in humans. In our previously published paper we have already summarized factors important for optimal bioavailability of synthesized compounds. ${ }^{52}$ According to the physico-chemical properties calculated by Molinspiration software tool, ${ }^{48}$ compounds $\mathbf{3 a}-\mathbf{h}$ and $3 \mathbf{s}$ fulfilled all requirements for good oral bioavailability, including $\mathrm{m}_{\mathrm{i}} \log \mathrm{P}<5, \mathrm{n}_{\mathrm{ON}}<10, \mathrm{n}_{\mathrm{OHNH}}<5$, $\mathrm{MW}<500$, TPSA $<140 \AA^{2}\left(<60 \AA^{2}\right.$ ), and $\mathrm{n}_{\text {rotb }}<10$ (Suplementary data, Table $\mathrm{S} 1)$.

\section{3. 2. Pharmacokinetic Properties of the Eugenyl Esters 3a-s}

Absorption properties of compounds $3 \mathbf{a}-\mathbf{s}$ were predicted by admetSAR ${ }^{49}$ (Supplementary data, Table S2). Based on the results, all of the studied compounds were predicted as compounds able to pass through blood-brain barrier and penetrate into the central nervous system, as compounds capable of being absorbed by intestine, as well as compounds permeable across Caco- 2 cells. Compounds 3g-r were predicted as P-glycoprotein substrates, while compound 3s was predicted as P-glycoprotein inhibitor. None of the studied compounds was predicted as inhibitor of renal organic cation transporter.

Metabolic properties of the eugenyl esters 3a-s were predicted by admetSAR ${ }^{49}$ (Supplementary data, Table S3). None of the studied compounds was predicted as CYP2C9 and/or CYP2D6 substrate, while 15 compounds (3c, 3e-r) were predicted as CYP3A4 substrate. Regarding CYP450 inhibition, enzyme CYP1A2 might be inhibited by compounds $3 \mathbf{c}, 3 \mathbf{e}-\mathbf{s}$, CYP2C 9 by compound 3s, CYP2C19 by compounds $\mathbf{3 b}, \mathbf{3 c}, \mathbf{3 h}-\mathbf{s}$. However, none of the compounds was predicted as CYP2D6 inhibitor. Most of the investigated compounds were predicted to have low CYP 450 inhibitory promiscuity, except compounds $\mathbf{3 a}, \mathbf{3} \mathbf{d}$ and $\mathbf{3 s}$.

\section{3. 3. Toxicological Properties of the Eugenyl Esters 3a-s}

Using Toxtree prediction tool based on a decision tree approach, ${ }^{51}$ we have predicted structural alerts for DNA and protein binding for the studied compounds. Regarding DNA binding alerts, all of the studied compounds were predicted to have the ability to undergo nucleophilic aliphatic substitution $\left(S_{N} 1\right.$ reactions) and Michael addition. Among the protein binding alerts, compounds $\mathbf{3 a}-\mathbf{s}$ were predicted as compounds able to undergo Michael addition and $\mathrm{S}_{\mathrm{N}} 2$ reactions; $3 \mathbf{a}-\mathbf{s}$ were predicted as compounds able to participate in acyl transfer (results given in Supplementary data, Tables S4 and S5).

Based on the toxicological properties predicted by admetSAR ${ }^{49}$ compounds $\mathbf{3 a}-\mathbf{s}$ have been characterized as weak hERG (human Ether-à-go-go-Related Gene) inhibitors, non-AMES toxic and non-carcinogenic, but highly toxic for fish, Tetrahymena pyriformis and honey bee. Five compounds ( $\mathbf{3} \mathbf{a}-\mathbf{c}, \mathbf{3 e}$ and $\mathbf{3 g}$ ) were predicted as ready biodegradable. According to the risk for acute oral toxicity, all of the studied compounds (3a-s) were predicted as Category III, or slightly toxic compounds, with $\mathrm{LD}_{50}$ values between 500 and $5000 \mathrm{mg} / \mathrm{kg}$. Depending on the median toxic dose $\left(\mathrm{TD}_{50}\right)$, all compounds were predicted as "non-required" or non-carcinogenic chemicals (Supplementary data, Table S6).

Toxicological properties of the studied compounds predicted by DataWarrior ${ }^{50}$ indicate that all compounds have high risk for tumorigenic and irritant effects. All compounds were predicted with no risk for mutagenic effects and no risk for reproductive effects (Supplementary data, Table S7).

\section{Conclusion}

We have synthesized nineteen esters of eugenol, of which four represent new compounds (31, 3n, 3p and 3r). All of the compounds were employed in antimicrobial bioassay, with several compounds tested for the first time in antibacterial (3b-d, $\mathbf{3 g}-\mathbf{r})$ and all of the compounds (3as) ever tested in antifungal activity bioassay. The results have indicated microorganisms' selective susceptibility, with B. subtilis (3d, 31, 3n-r), S. aureus (3e and $\mathbf{3 f}$ ), C. albicans (3d, 3f, 3n, 3r-s) and A. niger (3f, 3s) as the most susceptible ones, with minimal inhibitory or cidal concentrations (MIC/MBC/MFC) never exceeding the range of activity of eugenol as the parent compound. Based on current results and on our previously published ones, ${ }^{43}$ having in best case comparable but never greater MIC values than for parent phenolic compound we could not confirm results obtained by previous groups. ${ }^{29-31}$ Our results have shown the importance of free phenolic hydroxyl group in terms of activity against microbials, however more detailed research should be conducted involving also a more 
complex viewpoint, as for instance, the disclosure of mechanism of action of eugenyl esters on bacteria and fungi.

The results of our in silico study have shown that compounds fulfilling Lipinski's rule of five are $\mathbf{3 a}-\mathbf{h}$ and $\mathbf{3 s}$ and were predicted to have good oral bioavailability. All of the studied compounds were predicted as to be able to pass through blood-brain barrier capable of being absorbed by intestine and permeable across Caco-2 cells. Metabolic properties differ within the studied compounds, depending on whether they act as substrate and/or inhibitor of various CYP450 enzymes. Compounds $\mathbf{3 a}-\mathbf{s}$ were predicted as weak hERG inhibitors, non-AMES toxic and non-carcinogenic, but highly toxic for fish, T. pyriformis and honey bee. Further, they were predicted as Category III of risk for acute oral toxicity, as well as compounds with high risk for tumorigenic and irritant effects. Finally, they exhibited at least two structural alerts for both DNA and protein binding, indicating their ability to act as a mutagen or a genotoxic.

Taking in consideration predicted in silico properties and estimated drug likeness score, pharmacological and toxicological profile, eugenyl esters might be used as prodrugs of eugenol due to an expected enhanced lipophilicity ${ }^{53}$ and passive membrane transport.

\section{Acknowledgement}

The authors acknowledge the Ministry of Science and Technological Development of Serbia for financial support (projects 451-03-821/2012-14, 172044 and 172047) as well as internal project of Faculty of Medicine, University of Niš (project no. 4).

\section{References}

1. T. B. de Souza, M. Orlandi, L. F. L. Coelho, L. C. C. Malaquias, A. L. T. Dias, R. R. de Carvalho, N. C. Silva, D. T. Carvalho, Med. Chem. Res. 2014, 23, 496-502.

DOI:10.1007/s00044-013-0669-2

2. P. M. Dewick, Medicinal natural products: a biosynthetic approach, second edition, John Wiley \& Sons Ltd, Baffins Lane, Chichester, West Sussex, PO19 1UD, England, 2002.

3. T. Koeduka, E. Fridman, D. R. Gang, D. G. Vassão, B. L. Jackson, C. M. Kish, I. Orlova, S. M. Spassova, N. G. Lewis, J. P. Noel, T. J. Baiga, N. Dudareva, E. Pichersky, Proc. Natl. Acad. Sci. U. S. A. 2006, 103, 10128-10133.

DOI:10.1073/pnas.0603732103

4. H. Surburg, J. Panten, Common fragrances and flavor materials: preparation, properties and uses, $5^{\text {th }}$ edition, Wiley-VHC Verlag GmbH \& Co. KGaA, 2006.

DOI:10.1002/3527608214

5. T. S. Kaufman, J. Braz. Chem. Soc. 2015, 26, 1055-1085.

6. L. I. Paula-Freire, G. R. Molska, M. L. Andersen, E. L. Carlini, Planta Med. 2016, 82, 211-216.
7. S. A. Guenette, F. Beaudry, J. F. Marier, P. Vachon, J. Vet. Pharmacol. Therap. 2006, 29, 265-270.

DOI:10.1111/j.1365-2885.2006.00740.x

8. S. Lahloua, A. F. Figueiredo, P. J. C. Magalhaes, J. H. Leal-Cardoso, P. D. Gloria, Life Sci. 2004, 74, 2401-2412.

DOI:10.1016/j.lfs.2003.09.063

9. M. E. Hidalgo, C. de la Rosa, H. Carrasco, W. Cardona, C. Gallardo, L. Espinoza, Quim. Nova 2009, 32, 1467-1470.

DOI:10.1590/S0100-40422009000600020

10. I. Gülçin, J. Med. Food 2011, 14, 975-985. DOI:10.1089/jmf.2010.0197

11. M. d'Avila Farias, P. S. Oliveira, F. S. Dutra, T. J. Fernandes, C. M. de Pereira, S. Q. de Oliveira, F. M. Stefanello, C. L. Lencina, A. G. Barschak, J. Pharm. Pharmacol. 2014, 66, 733-746. DOI:10.1111/jphp.12197

12. A. N. Daniel, S. M. Sartoretto, G. Schmidt, S. M. Caparroz-Assef, C. A. Bersani-Amado, R. K. N. Cuman, Braz. J. Pharmacogn. 2009, 19, 212-217.

DOI:10.1590/S0102-695X2009000200006

13. H. Sadeghian, S. M. Seyedi, M. R. Saberi, Z. Arghiania, M. Riazia, Bioorg. Med. Chem. 2008, 16, 890-901.

DOI:10.1016/j.bmc.2007.10.016

14. M. Machado, A. M. Dinis, L. Salgueiro, J. B. A. Custodio, C. Cavaleiro, M. C. Sousa, Exp. Parasitol. 2011, 127, 732-739. DOI:10.1016/j.exppara.2011.01.011

15. T. Ueda-Nakamura, R. R. Mendonca-Filho, J. M. Morgado-Diaz, P. K. Maza, B. P. Dias Filho, D. A. G. Cortez, D. S. Alviano, M. S. S. Rosa, A. H. C. S. Lopes, C. S. Alviano, C. V. Nakamura, Parasitol. Int. 2006, 55, 10-99. DOI:10.1016/j.parint.2005.10.006

16. H. Kabuto, M. Tada, M. Kohno, Biol. Pharm. Bul. 2007, 30, 423-427. DOI:10.1248/bpb.30.423

17. D. P. Bezerra, G. C. G. Militão, M. C. de Morais, D. P. de Sousa, Nutrients 2017, 9, 1-15. DOI:10.3390/nu9121367

18. P. K. Awasthi, S. C. Dixit, N. Dixit, A. K. Sinha, J. Pharm. Res. 2008, 1, 215-220.

19. R. Ghosh, N. Nadiminty, J. E. Fitzpatrick, W. L. Alworth, T. J. Slaga, A. P. Kumar, J. Biol. Chem. 2005, 280, 5812-5819. DOI:10.1074/jbc.M411429200

20. L. Fangjun, Y. Zhijia, Thorac. Cancer 2018, 9, 25-29. DOI:10.1111/1759-7714.12508

21. A. Marchese, R. Barbieri, E. Coppo, I. E. Orhan, M. Daglia, S. F. Nabavi, M. Izadi, M. Abdollahi, S. M. Nabavi, M. Ajami, Crit. Rev. Microbiol. 2017, 43, 668-689. DOI:10.1080/1040841X.2017.1295225

22. D. R. Gang, J. Wang, N. Dudareva, K. H. Nam, J. E. Simon, E. Lewinsohn, E. Pichersky, Plant Physiol. 2001, 125, 539-555. DOI:10.1104/pp.125.2.539

23. K. Chaieb, T. Zmantar, R. Ksouri, H. Hajlaoui, K. Mahdouani, C. Abdelly, A. Bakhrouf, Mycoses 2007, 50, 403-406. DOI:10.1111/j.1439-0507.2007.01391.x

24. S. Hemaiswarya, M. Doble, Phytomedicine 2009, 16, 9971005. DOI:10.1016/j.phymed.2009.04.006

25. E. Pinto, L. Vale-Silva, C. Cavaleiro, L. Salgueiro, J. Med. Microbiol. 2009, 58, 1454-1462.

DOI:10.1099/jmm.0.010538-0 
26. I. A. Freires, C. Denny, B. Benso, S. M. de Alencar, P. L. Rosalen, Molecules 2015, 22, 7329-7358.

DOI:10.3390/molecules20047329

27. H. Carrasco, M. Raimondi, L. Svetaz, M. Di Liberto, M. V. Rodriguez, L. Espinoza, A. Madrid, S. Zacchino, Molecules 2012, 17, 1002-1024. DOI:10.3390/molecules 17011002

28. P. H. O. Abrao, R. B. Pizi, T. B. De Souza, N. C. Silva, A. M. Fregnan, F. N. Silva, L. F. L. Coelho, L. C. C. Malaquias, A. L. T. Dias, D. F. Dias, M. P. Veloso, D. T. Carvalho, Chem. Biol. Drug Des. 2015, 86, 459-465. DOI:10.1111/cbdd.12504

29. T. B. de Souza, K. M. de Oliveira Brito, N. C. Silva, R. P. Rocha, G. F. de Sousa, L. P. Duarte, L. F. L. Coelho, A. L. T. Dias, M. P. Veloso, D. T. Carvalho, D. F. Dias, Chem. Biol. Drug Des. 2016, 87, 83-90. DOI:10.1111/cbdd.12625

30. D. B. Resende, H. H. de Abreu Martins, T. B. de Souza, D. T. Carvalho, R. H. Piccoli, R. F. Schwan, D. R. Dias, Food Chem. 2017, 237, 1025-1029. DOI:10.1016/j.foodchem.2017.06.056

31. G. Zengin, Chem. Nat. Compd. 2011, 47, 550-555. DOI:10.1007/s10600-011-9994-1

32. V. Chiaradia, N. Paroul, R. L. Cansian, C. V. Júnior, M. R. Detofol, L. A. Lerin, J. Vladimir Oliveira, D. Oliveira, Appl. Biochem. Biotechnol. 2012, 168, 742-751.

DOI:10.1007/s12010-012-9814-5

33. N. S. Radulović, M. Z. Mladenović, P. D. Blagojević, Z. Z. Stojanović-Radić, T. Ilić-Tomić, L. Senerović, J. Nikodinović-Runić, Food Chem. Toxicol. 2013, 62, 554-565.

DOI:10.1016/j.fct.2013.09.017

34. D. P. Bezerra, G. C. Gadelha Militão, M. C. de Morais, D. P. de Sousa, Nutrients 2017, 9, pii: nu9121367.

DOI:10.3390/nu9121367

35. L. Kamadatu, M. Santoso, Synthesis and Cytotoxicity of 4-Allyl-2 Methoxyphenol Derivatives, http://iptek.its.ac.id/index. php/jps/article/view/1166/1026, (Accessed: March 10, 2018).

36. H. A. Carrasco, L. C. Espinoza, V. Cardile, C. Gallardo, W. Cardona, L. Lombardo, K. M. Catalán, M. F. Cuellar, A. Russo, J. Braz. Chem. Soc. 2008, 19, 543-548.

DOI:10.1590/S0103-50532008000300024

37. J. D. F. Barbosa, V. B. Silva, P. B. Alves, G. Gumina, R. L. C. Santos, D. P. Sousae, S. C. H. Cavalcanti, Pest Manag. Sci. 2012, 68, 1478-1483. DOI:10.1002/ps.3331
38. M. R. C. Raja, A. B. Velappan, D. Chellappan, J. Debnath, S. K. Mahapatra, Eur. J. Med. Chem. 2017, 139, 503-518.

DOI:10.1016/j.ejmech.2017.08.030

39. H. C. Brown, J. Am. Chem. Soc. 1938, 60, 1325-1328. DOI:10.1021/ja01273a014

40. W. G. Rose, J. Am. Chem. Soc. 1947, 69, 1384-1387. DOI:10.1021/ja01198a043

41. J. Paolini, A. Muselli, A. F. Bernardini, A. Bighelli, J. Casanova, J. Costa, Flavour Fragr. J. 2007, 22, 479-487.

DOI:10.1002/ffj.1824

42. D. Pavlović, B. Modec, D. Dolenc, Acta Chim. Slov. 2015, 62, 362-370. DOI:10.17344/acsi.2014.1306

43. J. Lazarević, A. Kolarević, A. Đorđević, G. Stojanović, A. Šmelcerović, P. Ciuffreda, E. Santaniello, Acta Chim. Slov. 2017, 64, 603-612. DOI:10.17344/acsi.2017.3356

44. A.E. Díaz-Alvarez, P. Crochet, V. Cadierno, Tetrahedron 2012, 68, 2611-2620. DOI:10.1016/j.tet.2012.01.083

45. H. Van den Dool, P. D. Kratz, J. Chromatogr. A 1963, 11, $463-$ 471. DOI:10.1016/S0021-9673(01)80947-X

46. NCCLS, Performance Standards for Antimicrobial Susceptibility Testing: Eleventh Informational Supplement, M100-S11. 2003, National Committee for Clinical Laboratory Standards, Wayne, PA.

47. S. A. Sarker, L. Nahar, Y. Kumarasamy, Methods 2007, 42, 321-324. DOI:10.1016/j.ymeth.2007.01.006

48. Molinspiration Home Page. http://www.molinspiration.com/ (Accessed January 2018).

49. admetSAR Home Page. http://lmmd.ecust.edu.cn:8000/predict/ (Accessed January 2018).

50. DataWarrior Home Page. http://www.openmolecules.org/datawarrior/ (Accessed January 2018).

51. G. Patlewicz, N. Jeliazkova, R. J. Safford, A. P. Worth, B. Aleksiev, Environ. Res. 2008, 19, 495-524.

52. C. A. Lipinski, F. Lombardo, B. W. Dominy, P. J. Feeney, $A d v$. Drug Deliv. Rev. 2012, 64, 4-17.

DOI:10.1016/j.addr.2012.09.019

53. D. Yancheva, E. Cherneva, M. Quick, B. Mikhova, B. Shivachev, R. Nikolova, A. Djordjevic, M. Untergehrer, G. Jürgenliemk, B. Kraus, A. Smelcerovic, Acta Chim. Slov. 2015, 62, 689-699. DOI:10.17344/acsi.2015.1418

\section{Povzetek}

Rezultati, predstavljeni v tem članku, so nadaljevanje naših študij modifikacij molekul naravnega izvora, s posebnim poudarkom na aciliranju fenolne funkcionalne skupine. Pripravili smo majhno, fokusirano knjižnico devetnajstih estrov evgenola, od katerih so štiri nove spojine. $\mathrm{Z}$ vsemi spojinami smo izvedli in vitro antimikrobno testiranje. $\mathrm{Z}$ in silico računskimi študijami smo napovedali fizikalno-kemijske, farmakokinetične in toksikološke lastnosti, kar daje dodatne podatke za boljše usmerjanje raziskav v prihodnosti. 